\title{
How We Type: Eye and Finger Movement Strategies in Mobile Typing
}

\author{
Xinhui Jiang ${ }^{1}$, Yang Li ${ }^{1}$, Jussi P.P. Jokinen ${ }^{2}$, Viet Ba Hirvola ${ }^{2}$, Antti Oulasvirta ${ }^{2,3}$, Xiangshi Ren ${ }^{1}$ \\ ${ }^{1}$ Center for Human-Engaged Computing, Kochi University of Technology, Japan \\ ${ }^{2}$ Department of Communications and Networking, Aalto University, Finland \\ ${ }^{3}$ Finnish Center for Artificial Intelligence (FCAI), Finland
}

\begin{abstract}
Relatively little is known about eye and finger movement in typing with mobile devices. Most prior studies of mobile typing rely on log data, while data on finger and eye movements in typing come from studies with physical keyboards. This paper presents new findings from a transcription task with mobile touchscreen devices. Movement strategies were found to emerge in response to sharing of visual attention: attention is needed for guiding finger movements and detecting typing errors. In contrast to typing on physical keyboards, visual attention is kept mostly on the virtual keyboard, and glances at the text display are associated with performance. When typing with two fingers, although users make more errors, they manage to detect and correct them more quickly. This explains part of the known superiority of two-thumb typing over one-finger typing. We release the extensive dataset on everyday typing on smartphones.
\end{abstract}

\section{Author Keywords}

Text input; mobile device; eye-hand coordination; eye movement; finger movement.

\section{CCS Concepts}

-Human-centered computing $\rightarrow$ Text input; Graphical user interfaces; Mobile phones;

\section{INTRODUCTION}

This paper presents new data of how people type on touchscreen devices. Present-day understanding on typing is rooted mainly in studies with physical keyboards [16, 33, 37, 48], which differ in a few important respects from touchscreen keyboards on handheld devices. The most obvious are size, hand postures, the role of the thumbs, and the lack of physical keyswitches. Everyday mobile typing is carried out on the move too. Little research exists on the implications of these factors for how people move their gaze and fingers, which is surprising, given the prevalence of these devices and general awareness that visual and manual strategies affect performance

Permission to make digital or hard copies of all or part of this work for personal or classroom use is granted without fee provided that copies are not made or distributed for profit or commercial advantage and that copies bear this notice and the full citation on the first page. Copyrights for components of this work owned by others than the author(s) must be honored. Abstracting with credit is permitted. To copy otherwise, or republish, to post on servers or to redistribute to lists, requires prior specific permission and/or a fee. Request permissions from permissions@ acm.org.

CHI '20, April 25-30, 2020, Honolulu, HI, USA.

(C) 2020 Copyright is held by the owner/author(s). Publication rights licensed to ACM ACM ISBN 978-1-4503-6708-0/20/04 ...\$15.00.

DOI: https://dx.doi.org/10.1145/3313831.3376711 across a plethora of skilled activities, such as typing on physical keyboards [16, 29], driving [35, 43], and gaming [20, 21].

Our goal is to understand movement strategies in mobile typing. Visuomotor strategies are learned over experience and coordinate manual and sensory actions like hand and eye movements [4]. They are associated with performance [53]. For typing, in general, a strategy is needed for coordinating the allocation of visual attention between the text display and the keyboard, and to guide the timing and speed of finger movements $[14,16]$. A typing strategy must also regulate the speed and accuracy of aiming, which should adapt, depending on target sizes and the permitted rate of errors $[7,22,25,30$, 56]. Prior research on mobile devices in particular suggests that sharing of the visual attention may have an important role. It must be devoted to guiding the fingers because of the lack of tactile landmarks $[9,57]$. At the same time, it may be needed for checking the correctness of the text and of predictions/corrections made by any intelligent text-entry method. Visual attention is required also in searching for rarely used characters on the keyboard [31, 57].

To identify and quantify movement strategies in mobile typing and their relationship to typing speed, one must have synchronized eye and finger movement data. Such data are needed for revealing movement strategies that are hard to report on verbally. Yet previous work on mobile typing has relied primarily on log data for touch events, which is not ideal for in-depth studies of visuomotor strategies. Hence, several questions are open. Firstly, is visual attention tightly coupled with finger movement? With physical keyboards, faster typists generally keep their attention on the monitor more than the keyboard $[16,29]$. One study of mobile devices with physical keyboards suggests that there are attention shifts between text display and the keyboard [24]; however, this phenomenon has not been described in detail for touchscreen typing. Secondly, what exactly initiates error correction? Do users detect errors directly after the erroneous keypress or much later? Thirdly, do strategies differ among common typing styles, such as onefinger and two-thumb typing? Two-thumb typing is known to be faster, with the gain attributed to rapid alternation between the lateral sides of the keyboard [45]. For answers, data on eye and finger movement are needed. High-quality datasets are also critical for efforts in predictive modelling and intelligent text entry methods. 

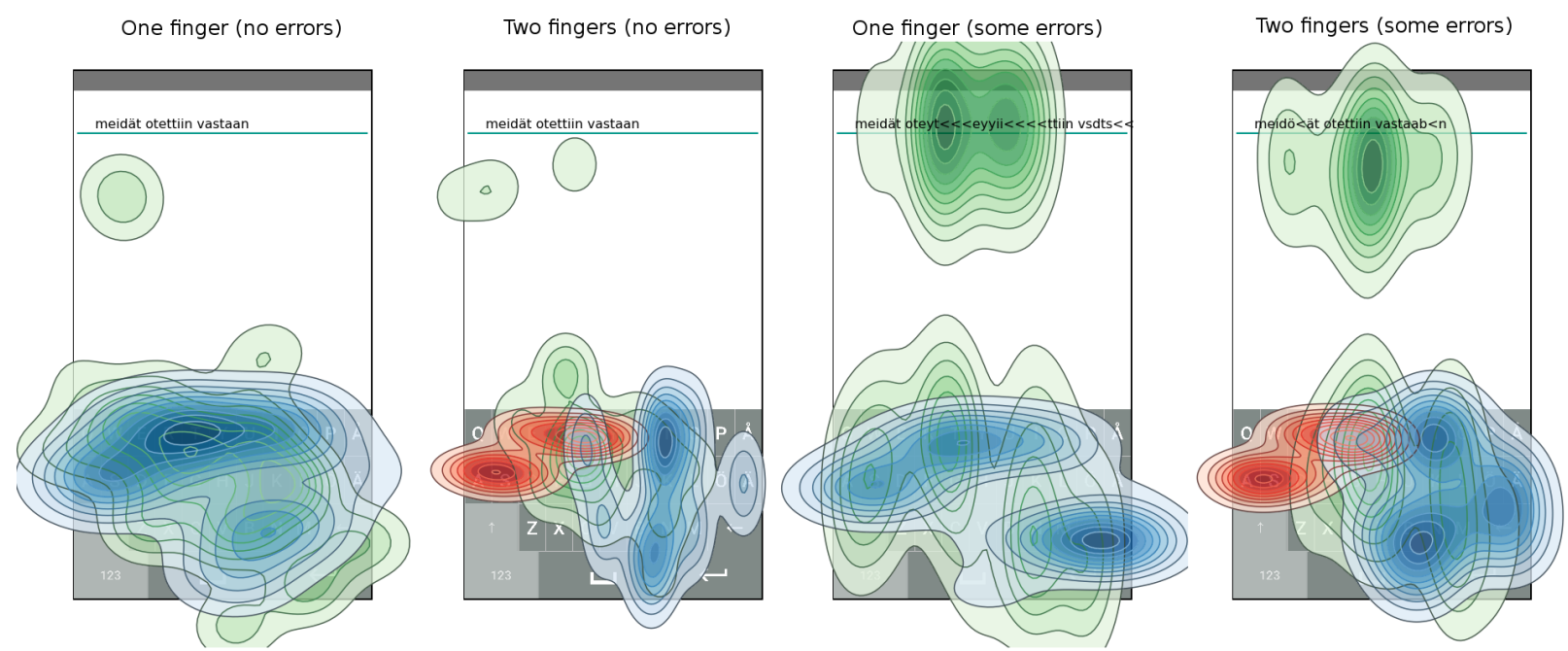

Figure 1. Illustration of our data as heatmaps of finger touchpoints (blue for right index finger or thumb, and red for left thumb) and eye movements (green). All present typing of the same sentence by a different participant: one (index) finger with no typing errors, two thumbs with no errors, one finger with errors, and two thumbs with errors. Glances at the text-entry area increase with the number of errors made, and error correction is visible as touches of Backspace. In two-thumb typing, visual guidance of the fingers is less in demand, so the gaze covers smaller areas of the keyboard.

Here, we report on findings from an exploratory study of transcription typing on a touchscreen device $(N=30)$, working with high-fidelity synchronized data from motion tracking, eye tracking, and on-device keypress logging. Our methodology for the study closely follows prior work on physical keyboard typing ("How We Type" [16]). To the best of our knowledge, the dataset presented here is the first of this type for mobile touchscreen devices. We report on eye movement, finger movement, eye-hand coordination, and predictors of typing performance with use of both one and two fingers for touchscreen devices. Figure 1 illustrates different glancing behaviors along with finger touch in our dataset, for a given sentence typed with one and two fingers and with and without errors. We devote the rest of the paper to reviewing today's understanding of movement strategies in mobile typing, then reporting on our method and results. While we report many detailed analyses, our overarching finding is that gazedeployment strategies are complex and much more important factors in typing performance than previously thought. We explain this and other findings in terms of how movement strategies adapt to the limited availability of visual attention. We also discuss the implications of the text entry system studied, which did not offer intelligent text entry techniques. The dataset is made publicly available.

\section{RELATED WORK}

Typing is a complex visuomotor process that engages multiple cognitive, perceptual, and motor abilities $[16,39,55]$. This behavior has been a topic of research for almost a century [13] and typing on touchscreen devices for three decades $[15,19$, 42]. Here, we discuss studies of physical and touchscreen typing, along with the contrasts they manifest. Papers on typing with a physical keyboard report average typing performance of around 50 words per minute (WPM) [14, 16]. Generally, typing speeds are lower for mobile devices, with reported averages between 36 and 41 WPM $[3,47]$.

\section{Typing with a Physical Keyboard}

Typing is a process carried out in phases, such as an input phase (grouping the to-be-typed text into chunks), parsing phase (decomposing the chunks into discrete characters), translation phase (converting characters into movement specifications), and execution phase (conducting the movements) [54, 55]. These phases are often interleaved, with the parallelism depending on a control hierarchy that is responsible for translating words into letters and motor plans [39]. Motor control strategies and ability affect typing performance. Expert typists can type quickly on account of automatic translation of letters into motor plans, which can be executed quickly [38]. This is associated with consistent finger-to-key mappings, which, along with preparation of the fingers, predict performance [16]. In addition, "rollover", wherein the next keypress is initiated while the previous key is still depressed, is prevalent among skilled typists especially; this improves typing performance [14]. Finally, alternating hands in typing of bigrams is generally superior to typing them with a single hand $[16,55]$.

Typing also requires visual attention. Pointing movements often consist of a rapid ballistic and a slower corrective movement. Generally, the eyes and the pointing hand demonstrate a "pointing synergy", both moving towards the target at the same time, with the eye arriving earlier due to large saccade speeds [26]. However, the tactile feedback provided by a physical keyboard permits attending to the text-entry area for the majority of the time, resulting in fast detection and correction of typing errors $[16,29]$. Typists who have studied touch typing and therefore have stable finger-to-key mappings do not have to glance down at the keyboard to search for keys [16, 48].

\section{Typing on Mobile Touchscreen Keyboards}

Touchscreen keyboards are generally much smaller than physical ones. Hence, most mobile typists use one or two fingers (generally thumbs) rather than the 3-9 fingers often used with physical keyboards $[16,47]$. In a pattern similar to physical 
keyboards', the use of two fingers (generally thumbs) yields faster typing, via finger alternation and preparatory movements of the free finger $[12,41,45]$. To overcome the slower overall typing on touschreen keyboards, several intelligent text-entry methods have been suggested $[34,47]$. These include auto-correction of mistyped words, prediction of the next word, dynamic resizing of keys, touchpoint correction, and accounting for hand posture. In a recent logging study of mobile typing, more than $80 \%$ of participants used some sort of intelligent text-entry aid [47]. In the interest of starting from a simpler visuo-motor-cognitive problem, we here focus on the non-aided case.

In addition to being smaller than physical keyboards, mobile touchscreen keyboards lack the tactile feedback of physical keys [23]. The fingers, lacking a physical reference point, need to be constantly monitored and guided by visual attention. Therefore, attention-sharing strategies differ between physicaland touschreen-keyboard typing. Glances at the text-entry area permit proofreading of the text entered but hinder visual guidance of the fingers, reducing typing speed [47]. However, undetected errors are costly; when detected later, mistakes require more steps and time to correct. Users are known to slow their typing in response to errors [5], and the strategic finger speed-accuracy tradeoff and proofreading frequency have a large impact on text-entry performance $[30,47,56]$.

Some studies have investigated the role of eye movements in non-typing touchscreen interactions. On internet-based tasks on tablets, gaze has been observed to precede touch with similar spatial and temporal features as observed with the mouse, but with individual differences $[60,61]$. Interaction between gaze and touch has also been utilized to adapt UIs with the help of a predictive model [49]. On tablets with split keyboards, it is often enough to attend only the text entry area and use peripheral vision to guide fingers [40].

\section{Theories and Models}

Typing models make predictions such as transcription time, inter-key-interval, and number of errors. Work thus far has focused mainly on modeling typing on physical keyboards, with fewer attempts relevant for mobile typing. Arguably the most popular statistical models are based on Fitts' law, which models aimed movement performance [17]. After calibration of its empirical parameters to touchscreen pointing, it can predict performance over a range of layout conditions [7]. However, this family of models can only approximate the skilled typing behavior achievable after extensive practice [62]. Absent from these models are the adaptive strategies that govern the distribution of visual attention and describe the visual guidance of finger and proofreading activity or the frequency of proofreading [5, 30, 31, 50, 56, 57].

Keystroke-Level Models (KLMs) break task execution into operations, such as recall, pointing, homing, and attention shifts [24]. However, being sequential models, KLMs do not cover parallel movement, learning, or the role of attention. Nor do they predict how the interface or the user's abilities affect the choice of typing strategies. Finally, simulation models are step-by-step programs emulating the cognitive and physical steps involved. One recent model covers 12 operations or production rules: creating a mental representation of the task, visually attending the target, pointing at the target, confirming that the task is done, etc. [9]. The operations are simulated with a cognitive architecture, which computes their execution times and links together the separate cognitive modules, such as memory and attention. Predictions can be generated for a wide range of task conditions. The model predicts how typing performance is influenced by, for instance, changes in the number of keys or in features such as their size.

The choice of movement strategy is very difficult to model with production rule-based cognitive architectures due to the sheer number of possible strategies. Recent research has turned to computational rationality $[18,36]$ to simulate strategic adaptation of gaze to the task environment $[31,56]$. There, typing performance is modeled as an adaptation of eye and finger movement to the constraints of the human visuomotor system and the interface. At the moment, however, this class of models does not fully cover typing phenomena, including parallel finger and eye movement, one hindrance having been the absence of a rich dataset.

\section{METHOD}

We designed an experiment to obtain a rich dataset of movement strategies in a transcription task. Participants typed representative everyday messages and were instructed to correct typing errors. This is consistent with other research, where the instruction is often to type "quickly and accurately" [14, 47] or to correct errors upon noticing them [16]. We collected data for typing with the index finger and with two thumbs, the most common typing styles [47]. We used a Qwerty keyboard without intelligent typing aids to establish a dataset of baseline typing phenomenon. All data were synchronized in time, and all positions were registered in a single coordinate system.

\section{Participants}

We recruited 33 subjects. Because of gaze-data loss (device error), the number of participants decreased to $N=30$ (18 female; age range $18-45, M=25.5, S D=5.9)$. Three participants were left-handed ( 1 female). All participants were native Finnish-speakers and had normal or corrected vision (correction strength between -4 and +4 ). All reported using Finnish in their typing, with most using computers (desktop or laptop) several times a day (two reported using only a few times a month). Also, most used touchscreen devices (mobile phones or tablets) several times a day (one reported once-aday use). The participants reported spending, on average, 16.7 hours $(S D=16.4)$ a week typing on a physical keyboard and 11.6 hours $(S D=8.0)$ on a mobile software keyboard. In our study, we observed typing performance of between 14.9 and 58.4 WPM for two-thumb typing and 19.1-33.3 WPM for one-finger typing. Each participant was compensated with two movie tickets (total worth about $€ 20$ ) for their time.

\section{Experiment Design}

Each subject typed 40 sentences randomly selected from a set of 75. There were 20 sentences (trials) each for one- and twofinger typing, with each participant typing in both conditions (order was counter-balanced). No participant was given the same sentence twice. 

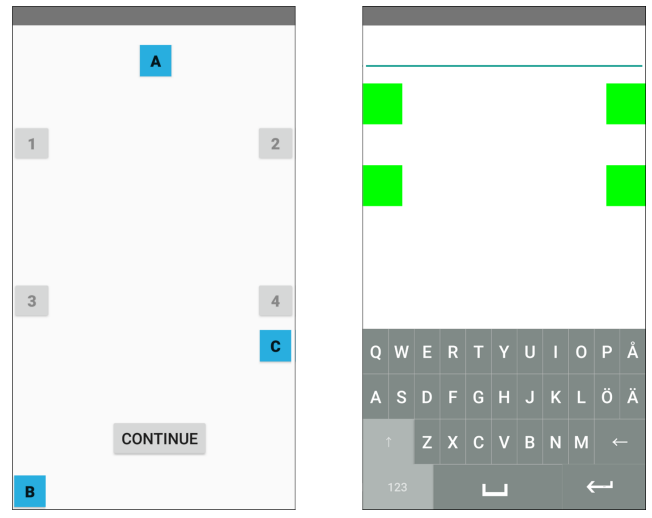

Figure 2. Left: A view for calibrating the eye tracker. Right: User interface of the typing task. The green boxes are for eye-tracking purposes.

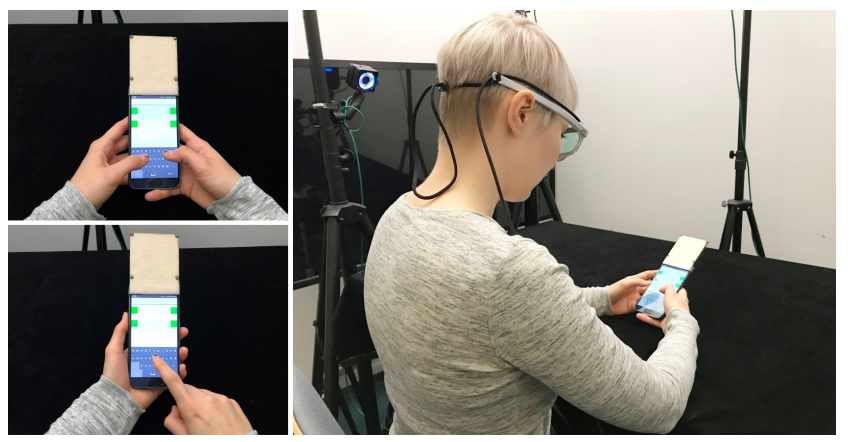

Figure 3. Posture for holding the device and sitting during the experiment. Shown are grips for one- and two-finger typing. The block above the device is for tracking the phone position.

\section{Materials}

Smartphones with a 4.7-5.5-inch touchscreen form the mainstream of the current market [28]. From among those devices we chose the Samsung Galaxy S6 smartphone $(1440 \times 2560$, $577 \mathrm{ppi}$ ) with a screen size of 5.1 inches. We developed a custom typing application for collecting key-pressing data and permitting the synchronization of data sources. The typing application is shown in Figure 2. Its two main views were the calibration view and the typing view, the former used for synchronizing data sources at the start of the task block and the latter for the transcription tasks themselves. The keyboard in the application had a standard Finnish Qwerty layout (key height: $10.06 \mathrm{~mm}$ ). The participants transcribed relatively simple, memorable everyday sentences, selected from the Enron Mobile Email Database [16, 59]. Seventy-five sentences were translated into Finnish by a native speaker and checked by one of the authors. All sentences were stripped of special characters and punctuation, and everything was in lowercase. Mean sentence length was 20 characters $(S D=4)$.

\section{Procedure}

Firstly, participants were told that the purpose of the study was to analyze the movement of the eyes and fingers in smartphone typing, and they filled in a background questionnaire. During the experiment, they sat in a chair at an adjustable-height table with the smartphone freely in their hands, which were resting on the table (see Figure 3). They were then given five minutes to practice and become familiar with the typing interface. After three-point calibration for eye-tracking glasses, participants were asked to press four buttons on the screen, marked with the numbers 1 to 4 , in ascending order, for synchronization between the motion tracker and smartphone. Each task trial consisted of one sentence, which was given to the participant aurally via a speech synthesizer to avoid unnecessary eye movement during the experiment. The participants were asked to repeat the sentence aloud to confirm that it was heard correctly and to strengthen the memory of the sentence, after which they could start typing. They were asked to type as quickly as possible and not leave errors in the final sentence submitted. As the task block dictated, the participant used either two thumbs (two-finger condition) or the index finger of the dominant hand (one-finger condition). For two-finger typing, participants were asked to hold the device in both hands and perform typing with two thumbs. For one-finger typing, they were asked to type with only the index finger of their dominant hand and hold the smartphone in the other hand. During the experiment, we suggested that the participants rest their arms on the table and try to keep the same posture throughout the experiment block [10]. However, there were no physical constraints to movement, and the subjects reported no discomfort. Error correction could be performed via a backspace button, with no other means provided, such as moving the cursor by touching the typed text. The trial time for one sentence was calculated as the time from the first keypress to pressing Enter, keypress being defined as the moment of a keydown log event.

\section{Data Collection and Preprocessing}

We collected three types of data: eye movement, finger motion, and keypresses. For eye movements, we used SMI model $2 \mathrm{~W}$ $A$ eye-tracking glasses ( $60 \mathrm{~Hz}$ at $30 \mathrm{FPS})$. The glasses had infrared cameras tracking eye movements and a forward field camera to record the screen of the mobile device held in the hands. Participants with corrected vision had corresponding corrective lenses attached. The three-point calibration was done via the calibration screen (on the left in Figure 2), with the participant asked to focus on the blue rectangles one at a time. In the experiment proper, the green rectangles (in the right pane of Figure 2) were used to transform the eye-tracking coordinates into device screen coordinates.

To track finger movement, we used an OptiTrack Prime 13 motion-capture system that provides $3 \mathrm{D}$ precision of up to $0.2 \mathrm{~mm}$ at close proximity. In one-finger typing, a reflective marker was attached to the top-middle part of the nail of the index finger of the dominant hand; in two two-thumb typing, one was attached to each thumb. The system was calibrated at the start of the block, with the same calibration screen as for the eye-tracking device (see Figure 2); the participants were asked to type the numbered blocks in order. For turning the finger position into device coordinates, four reflective markers were placed above the smartphone, in a holder (see Figure 3).

We checked all data manually and excluded three participants because of loss of fixation data (resulting in $N=30$ ). From the remaining participants' data, 244 trials out of the 1,199 
were excluded from eye-movement-related analyses due to data corruption (i.e., fixation data were present for less than $90 \%$ of the trial). The loss was not correlated with sentence length ( $M=20.44$ words before data removal, $M=20.46$ after). Loss of some motion-tracking data led to 45 further trials being excluded from finger-movement-related analyses (same criterion; also no change in sentence length). Finger tracking data were validated by confirming that the lowest local points of the finger(s) coincided with the pressing of keys in the device log. We extracted the coordinates from the raw data on finger and eye movements and converted them into a common coordinate system for the smartphone screen. In the data, the upper-left corner of the screen is the origin $(0,0,0)$, with $x$ axis values increasing toward the right of the device and $y$ values from top to bottom. The distance from the screen facing upward is the positive $z$ value. The unit in a datum refers to one pixel of the smartphone screen. The motiontracking system labeled and tracked each marker during the experiment. In the two-thumb typing condition, in cases where the tracker confused the fingers with each other due to their close proximity, we checked and corrected the data manually.

\section{Metrics}

We followed the guidance for typing performance metrics [64]. For eye and finger movement data, we compare the metrics to previous eye-and-finger-tracking study of physical keyboard typing [16]. The metrics used can be summarized thus:

- Inter-key interval (IKI) [16]: time between two subsequent keypresses.

- Words per minute (WPM) [16]: the number of standard words (every five characters in the final input text) divided by the time spent on typing.

- Backspace [47]: the number of Backspace presses during typing of a sentence.

- Uncorrected error rate [64]: non-corrected incorrect keystrokes as a percentage of the sum of incorrect (whether fixed later or not) and correct keystrokes.

- Corrected error rate [64]: incorrect but rectified keystrokes as a percentage of the above sum.

- Immediate error correction [2]: the frequency of error correction in which the user immediately identified and corrected an error with a subsequent Backspace press.

- Delayed error correction [2]: the frequency of error correction wherein the user tried to correct previously missed errors in the middle of the input stream.

- Chunk length: the average length of a chunk during typing of each sentence. In typing, "chunking" refers to splitting the sentence into smaller pieces to manage working memory load [1]. We identified the border of a chunk with when a clear increase in IKI is observed $[11,65]$. The difference between neighboring IKIs is denoted as IKI difference (IKID). If the difference between neighboring IKIs is greater than the average IKID for the sentence, the key-pressing moment is considered to be a chunk border.

- Gaze shift: the average number of glances away from the keyboard area into the text area during typing of a sentence. The areas are defined as either the text area or the keyboard, both extended by $1.40 \mathrm{~cm}$ to all directions to account for foveal vision and possible slight drift in the eye tracking data. Gaze shift has previously been measured as the number of gaze shifts from the monitor to the keyboard, reflecting most of the attention being put on the monitor [16]. We measured it in the opposite way, assuming that most of the attention would be on the touchscreen keyboard.

- Time ratio for gaze on keyboard: the percentage of the time spent glancing at the keyboard. This is obtained by dividing the duration of gazing at the keyboard area by total trial time [16].

- Entropy [16]: how consistently a key is pressed by the same finger in the two-finger typing condition. For each key $k$, given a frequency distribution over the two fingers, we compute the entropy as $H_{k}=-\sum_{f \in \text { Fingers }} p_{f} \log _{2}\left(p_{f}\right)$, where $p_{f}$ is the probability of finger $f$ pressing key $k$. The average entropy of a finger-to-key mapping is then computed as a sum over the entropy of each key weighted by the frequency of the corresponding letter. If a given key is always pressed by the same finger and this is true for all keys, the entropy is 0 . To represent the finger-key mapping graphically, we show the distribution of touchpoints, using different colors for different fingers. Heatmaps were created on the background of a keyboard screenshot with layers of density plotted via the SEABORN.KDEPLOT tool.

- Keys per finger [16]: the number of keys controlled by each thumb in the two-finger typing condition.

- Finger path: the distance that a finger has traveled during typing of a sentence.

- Distance to the next key [16]: at the moment of the current key-pressing, the average distance between the next target key and the finger for pressing that key.

- Finger alternation [16]: the percentage of bigrams entered with finger alternation.

- Same finger bigram [16]: the percentage of bigrams entered with the same finger.

- Letter repetition [16]: the percentage of pressed keys that are the same as the previous key.

Statistical tests were carried out using the Wilcoxon signedrank test with $\alpha=0.05$. Correlations between factors were calculated via Linear mixed-effects models with the LME4 package for R. Below, we report standardized $\beta \mathrm{s}$ as the correlation metric, noting any control variables that were used. In addition, all models had the task condition (number of fingers used) as a fixed effect, and subject and sentence-level as random effects [32]. The $p$-values for $\beta$ estimates were calculated via Satterthwaite approximation to degrees of freedom.

\section{RESULTS}

We collected, in total, 31,988 keypresses from the 30 participants (16,593 in the two-finger and 15,395 in the one-finger condition). Table 1 summarizes our main findings, aggregated first at subject level and then on grand condition level.

\section{Typing Performance}

As expected, we found statistically significant differences in typing performance between two-finger and one-finger typing 


\begin{tabular}{|c|c|c|c|c|c|c|c|}
\hline & \multirow[b]{2}{*}{ Measure } & \multicolumn{2}{|c|}{ Two-finger } & \multicolumn{2}{|c|}{ One-finger } & \multicolumn{2}{|c|}{ Wilcoxon test } \\
\hline & & $\mathrm{M}$ & SD & M & SD & W (29) & \\
\hline \multirow{8}{*}{ Performance } & IKI (ms) & 266.81 & 63.56 & 380.94 & 50.95 & $82 * * *$ & -1.98 \\
\hline & WPM & 39.33 & 10.3 & 27.19 & 3.61 & $779 * * *$ & 1.57 \\
\hline & Backspace & 3.58 & 2.8 & 2.61 & 1.81 & 575.5 & $(0.41)$ \\
\hline & Uncorrected error rate (\%) & 0.6 & 0.87 & 0.56 & 0.71 & 468.5 & $(0.05)$ \\
\hline & Corrected error rate $(\%)$ & 12.23 & 7.29 & 9.38 & 5.75 & $586^{*}$ & 0.43 \\
\hline & Immediate error correction & 0.41 & 0.38 & 0.40 & 0.26 & 413 & $(0.03)$ \\
\hline & Delayed error correction & 0.93 & 0.83 & 0.63 & 0.47 & 569.5 & $(0.44)$ \\
\hline & Chunk length & 4.43 & 0.53 & 3.98 & 0.41 & $689 * * *$ & 0.94 \\
\hline \multirow{5}{*}{ Eye gaze } & Number of fixations & 18.79 & 8.05 & 24.04 & 4.56 & $192^{* * * *}$ & -0.81 \\
\hline & Fixation duration & 315.27 & 67.65 & 303.99 & 45.72 & 454 & $(0.2)$ \\
\hline & Saccade length $(\mathrm{cm})$ & 3.37 & 0.75 & 3.58 & 0.68 & 339 & $(-0.29)$ \\
\hline & Gaze shift & 3.4 & 2.31 & 3.91 & 1.5 & $269 *$ & -0.26 \\
\hline & Time ratio for gaze on keyboard & 0.6 & 0.16 & 0.7 & 0.14 & $263 * *$ & -0.69 \\
\hline \multirow{12}{*}{$\begin{array}{l}\text { Finger } \\
\text { movement }\end{array}$} & Entropy & 0.07 & 0.04 & 0 & 0 & - & - \\
\hline & Keys per fing & 4 & 0.68 & & \multirow[b]{2}{*}{0.55} & $0 * * *$ & -13.75 \\
\hline & Keys per finger right & 9.24 & 0.74 & & & $0 * * *$ & \\
\hline & Finger path left $(\mathrm{cm})$ & 23.06 & 2.82 & \multirow{2}{*}{25.29} & \multirow{2}{*}{1.33} & $179 * * *$ & -1.02 \\
\hline & Finger path right $(\mathrm{cm})$ & 26.16 & 2.11 & & & $702 * * *$ & 0.49 \\
\hline & Dist. to next key ${ }^{1}(\mathrm{~cm})$ & 1.2 & 0.15 & 2.3 & 0.1 & $0 * * *$ & -8.78 \\
\hline & Finger alternation (\%) & 39.91 & 4.59 & 0 & 0 & - & - \\
\hline & - IKI (ms) & 243.10 & 73.63 & - & - & - & - \\
\hline & Same finger bigram (\%) & 60.09 & 4.59 & 100 & 0 & $0 * * *$ & -12.30 \\
\hline & - IKI (ms) & 289.43 & 62.66 & 364.81 & 48.14 & $151^{* * * *}$ & -1.35 \\
\hline & Letter repetitio & 11.59 & 3.1 & 10.17 & 2.37 & $591 *$ & 0.51 \\
\hline & - IKI (ms) & 177.78 & 23.81 & 182.15 & 25.25 & 417 & $(-0.18)$ \\
\hline
\end{tabular}

Table 1. Overview of the results for the one- and two-finger typing conditions. Differences between the conditions are tested using the Wilcoxon Signedrank test $(d f=29)$, with effect size $d$ computed as Cohen's d value. ${ }^{1}$ Not including consecutive clicks on the same key. $\left.\left.\left.*\right) p<0.05 * *\right) p<0.01, * * *\right)$ $p<0.001$.

(all test results are in Table 1). Users made more errors when typing with two fingers than with one (corrected error rate $M=12.23 \%$ vs $M=9.38 \%$ ), although they corrected most of these before submitting the final sentence (the uncorrected error rates were $M=0.6 \%$ and $M=0.56 \%$ respectively). When using two fingers, participants were faster at typing (visible in lower IKI and higher WPM values), used longer chunks $(M=4.43$ vs. $M=3.98)$, and made fewer gaze shifts between the keyboard and the text-entry area. The chunk sizes identified here are in line with the morphology of the Finnish language [44]. The comparisons between one- and two-finger typing are consistent with the previously observed error rates of $10.80 \%$ and $8.17 \%$, and with typing speeds of 50.03 WPM and 36.34 WPM for two- and one-finger typing, respectively (the study cited did not include error correction) [3]. Participants exhibited more delayed error corrections than immediate error corrections, both in one- and in two-finger typing, meaning that most errors were detected only after typing of further characters.

We also correlated typing performance with background factors. Younger users were more likely to type more quickly and made less gaze shifts [47]. WPM values were negatively correlated with age $(\beta=-0.27, b=-0.64, p<.001)$ while gaze shift had a positive correlation with age $(\beta=0.33, p<.01)$. We did not observe a correlation between error rate and age.

\section{Finger Movement}

\section{Global finger movement}

Global finger movement is the length of the total travel path of a finger or fingers in a sentence. We found significant differences in path length between both left- and right-hand data from two-finger typing and data for the dominant hand in one-finger typing. The average finger path in one-finger use $(M=25.29 \mathrm{~cm})$ is shorter than the sum of the finger paths in two-finger use $(M=49.22 \mathrm{~cm})$. Two fingers together travel more during typing than one finger, because each is free to move while the other finger is typing. As we show below, this is likely to be related to preparation of the free finger.

\section{Finger-to-key mapping}

Work based on logging data has assumed that the left and right thumb split the keyboard area [45]. We revisited that assumption in light of the motion-tracking data. We found that, overall, the right thumb $(M=9.24)$ is in charge of more keys than the left $(M=4.00)$. The right hand covers a larger area during typing than the left does, as Figure 4 shows. We observed no significant effect of finger-to-key mapping entropy on typing speed or error rate, meaning that this choice of strategy did not influence the participants' performance much. The reason could be that, since visual attention is needed for guiding the fingers, the finger that presses the next key can be selected opportunistically. 

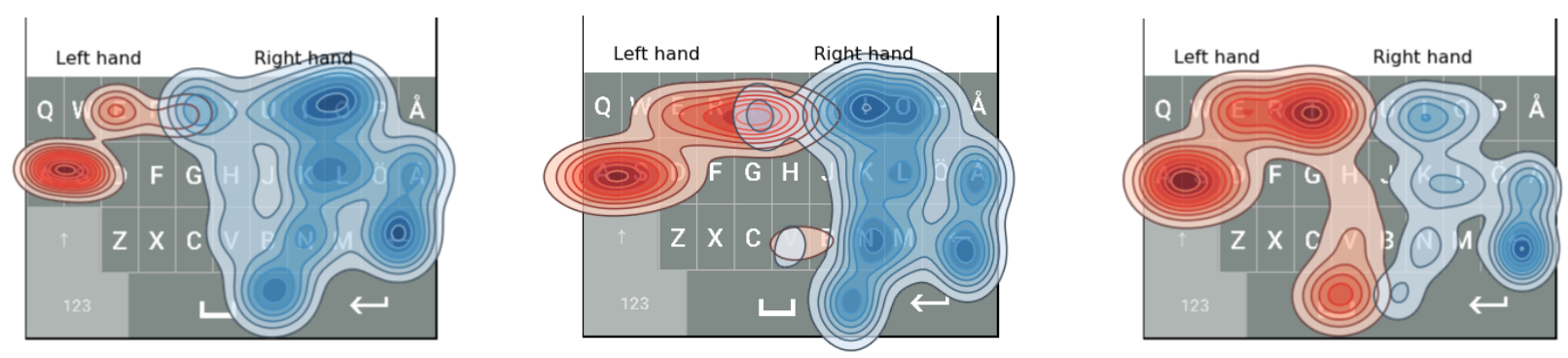

Figure 4. Heatmap showing finger-to-key mapping in two-thumb touch data of three participants (all sentences aggregated). Left thumb is red, right is blue. These patterns are representative of a tendency we found in the data for the right thumb to cover more keys than the left. The right hand was the dominant hand for most of the participants, but the same pattern was observed also for the left-handed participants.

\section{Finger preparation}

Finger preparation is an index of how much a finger moves in advance even before its "turn". It is measured as the distance of a finger from the key that it will press at the moment when the previous key is being pressed. We observed shorter preparation distance for two-thumb typing $(M=1.20 \mathrm{~cm})$ than for one-finger typing $(M=2.30 \mathrm{~cm})$. Analyzing this further, we found a negative correlation between distance to the next key and WPM, $\beta=-0.41, p<.001$, even when controlling for the true distance between subsequent keys (in the two-finger condition, this refers to subsequent keys pressed by the same finger). As is visible in Figure 5, the effect is similar between the one- and two-finger conditions, although, understandably, the latter condition allows more flexibility for preparing the finger that is not currently typing. In one-finger typing, the keypresses are executed with the finger in a sequential manner, so the distance between the finger and the next key is approximately equal to the inter-key distance. However, in the case of two-finger typing, users are free to decide and can control their fingers for parallel input with the two fingers. As one finger is clicking on a key, the other finger is already activated for aiming for the next key. The parallel control visibly increased the typing speed in the two-finger condition.

\section{Finger alternation}

Confirming previous findings based on log data $[45,47]$, we found a benefit for finger alternation. We observed a lower IKI in alternating ( $I K I=243.10 \mathrm{~ms})$ as opposed to using a single finger $(I K I=289.43 \mathrm{~ms})$. This benefit notwithstanding, it was more common to continue using the same finger: $60.09 \%$ of bigrams were typed with one finger instead of two. Completing a bigram with one finger was faster in two-thumb typing than in one-finger typing $(I K I=364.81 \mathrm{~ms})$. This can be attributed to the longer average travel distance when one finger is used in typing. Figure 6 shows the IKI distribution between types of bigrams for the same finger and alternating fingers in two-thumb typing, the same finger in one-finger typing, and a repeated letter in both two- and one-finger typing.

\section{Eye-hand Coordination}

\section{Eye-hand distance}

We examined the distance between the fixation point and the finger in one-finger typing (this cannot be unambiguously computed for the two-thumb case), so as to understand whether a closer eye-hand coupling can lead to better performance. We found a significant positive correlation between the average eye-hand distance and the average corrected error rate per sentence $(\beta=0.32, p<.01)$, controlling for the number of backspaces per sentence. This illustrates that typing errors are correlated with more visual attention on text area, which results in looser eye-hand coupling. For typing speed, as expected, we found a negative correlation between average eye-hand distance and average WPM per sentence (see Figure 7). However, this result may be explained by slower typists having to look at the text display more, which manifests itself here in long eye-hand distances. Therefore, we looked at eye-hand following specifically when both are operating in the keyboard area.

\section{Eye-hand following}

When pressing a key or in searching for one, the finger may follow the eye. To look at eye-hand following behavior, we extracted finger and eye movement paths in one-finger typing where the eyes stay in the keyboard area. We examined the dissimilarity between the finger movement and eye movement path by means of the Partial Curve Mapping (PCM) method, which uses a combination of arc length and area to determine the similarity between curves $[63,27]$. We found a positive correlation between WPM and dissimilarity, $\beta=0.16, p<$ .001. If a user types more quickly, there is less similarity. One explanation might be that fast typists have less need to guide their fingers with the eyes and so retain global supervisory control over the keyboard while trusting in the accuracy of their fingers. Also, we found a negative correlation between time ratio for looking at the keyboard and dissimilarity $(\beta=$ $-0.2, p<.001$ ), indicating, as expected, that the more the gaze is on the keyboard, the greater the similarity between the finger path and eye movement path.

More detailed investigation of eye and hand movement might help to explain this finding. Figure 8 shows the distance of the eye and the finger from the next key that is typed, taken from two partial example sentences from two participants (one-finger typing). Glances at the text-entry area are visible as large distances between the eye and the target key. In both sentences the finger and the eye move simultaneously toward the target key. The finger moves rapidly at first, and it slows down near the target for the final "peck". However, one can see a subtle difference between these participants: in the lower pane, the eye quickly finds the target key, after which 
it starts moving away from it even before the finger can peck it. Similarly, when backspacing, the upper-pane participant uses the eyes to locate Backspace whereas the lower-pane one looks at the text-entry area after having already visually located Backspace. It is possible that participants who trust their pointing accuracy more can free their vision for other tasks than guiding the finger, producing both faster typing and greater dissimilarity between the eye and finger paths.

\section{Proofreading and Error Correction}

\section{Gaze-shifting}

We defined a gaze shift as a glance from the keyboard at the text area, and we take a gaze shift to indicate either (preemptive) proofreading or error-correction activity: a glance is initiated to check the typed text for errors, attend it for possible new errors, or to control backspacing when an error has been found. Again, in our study, the only way for participants to correct errors was by using the Backspace button. The left pane in Figure 9 illustrates the average number of gaze shifts with one- and two-finger typing, and for both sentences that contained error correction and those that did not. In sentences with typing errors (and the subsequent error correction), our participants shifted gaze between the text-entry area and the keyboard more than in error-free sentences. The pattern is identical between the typing conditions, although the one-finger condition displayed slightly more gaze shifts.

More total time was used for looking at the keyboard in onefinger than in two-finger typing, and more was used for sentences without backspacing (right pane in Figure 9). This means that glancing behavior is more erratic under one-finger typing, with gaze shifts as well as less relative time spent glancing at the text area. Analyzing the correlation between the time ratio for keyboard glances and the corrected error rate, when controlling for uncorrected error rate, we observed a negative $\beta=-0.35, p<.001$. Similarly, the number of glances at the text-entry area correlates with the number of corrected errors, $\beta=0.58, p<.001$. Nevertheless, there were still, on average, 2.4 glances into the text-entry area for sentences that contained no error correction.

To investigate the impact of proofreading activity on typing performance further, we analyzed the correlation between WPM and gaze shifts, observing $\beta=-0.51, p<.001$. This

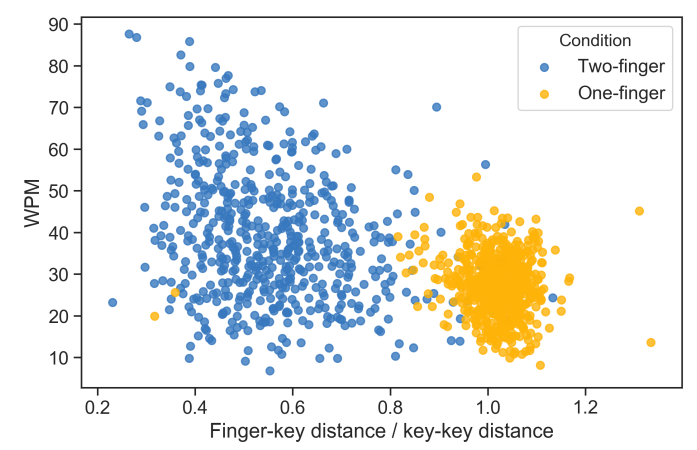

Figure 5. The impact of finger preparation on WPM by the task condition. The $x$ axis shows distance of finger from its next key, divided by the distance of the current and the next key (pressed by the same finger).

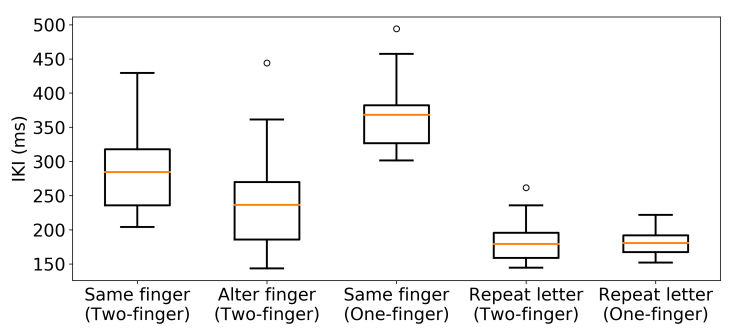

Figure 6. The impact of finger alternation for inter-key-intervals between different bigram types.

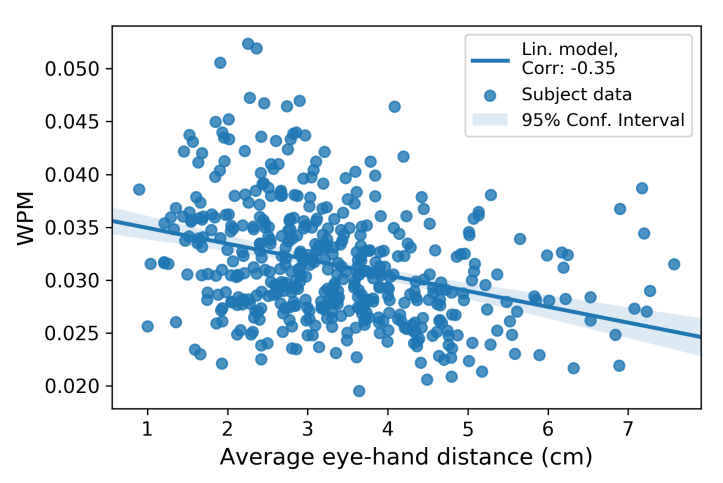

Figure 7. Eye-hand distance and average IKI per sentence.

negative correlation remained even after controlling for the amount of error correction the participants did (although the corrected estimate was smaller, $\beta=-0.17, p<.001$ ). Even for the subset of the data with only sentences containing no backspacing or uncorrected errors, we observed a negative correlation $\beta=-0.16, p<.001$. These findings mean that, irrespective of the number of errors made and corrected, typing performance is negatively correlated with gaze shifting between the keyboard and the text entry area. Reflecting on the same phenomenon, the percentage of sentence-typing time for which the eyes were on the keyboard had a small but statistically significant correlation with WPM, $\beta=0.11, p<.001$. Typists who focus more on the keyboard can reach higher textentry rates. This focus may reflect a typist's level of confidence in not having made typing errors.

\section{Correction of errors}

We looked at two types of error correction: immediate error correction refers to when the user immediately identifies an error and corrects it with a subsequent Backspace press; delayed error correction occurs when the user attempts to correct an error in the middle of the input stream that was missed or overlooked, via multiple Backspace presses. To investigate the latter error type further, we split consecutive Backspace presses into the first press, intermediate backspacing, and the final press of Backspace.

As shown in Figure 10, it took more time for the participants to press Backspace a single time or make the first of multiple backspaces, relative to an average keypress. For intermediate 


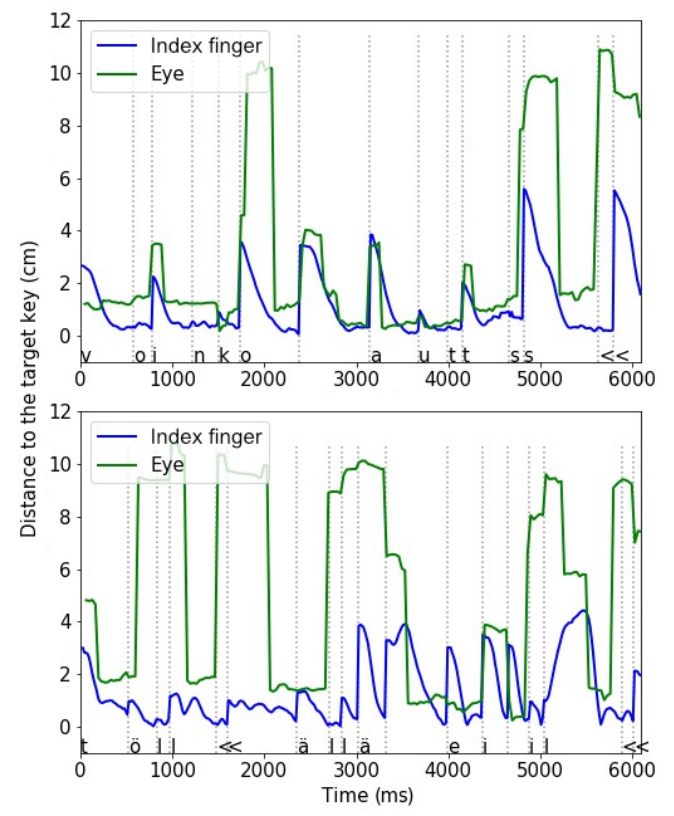

Figure 8. Key-by-key distance of the eyes and the finger in two partial sentences (truncated to about 6 seconds of typing; ' $<$ ' refers to Backspace). Note the different sentence in these examples.
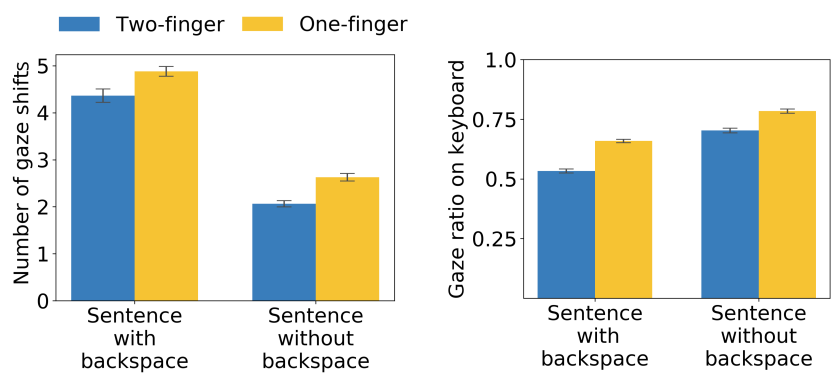

Figure 9. Left: Number of gaze shifts to text area. Right: Ratio of gaze spent looking at the keyboard. Error bars are standard errors.

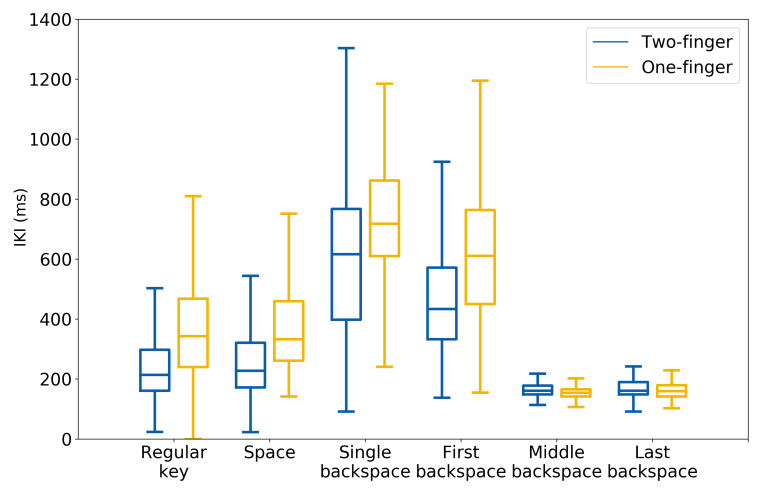

Figure 10. Typing interval for various types of key pressing

presses during a run of backspaces and for the final Backspace press, the average time consumed was much lower than that for an average keypress. The average time used for a single backspace was lower in typing with two fingers than in typing with one finger.

We calculated the frequency of various types of errorcorrection behavior across conditions. For two-thumb typing, more delayed error corrections $(M=0.93)$ were observed than immediate ones $(M=0.41)$. Finally, we investigated the connection between typing errors on typing speed. Since errors and the backspacing that erases them (along with any correctly typed text between the error and the correction) do not contribute to typing the sentence, we expected a higher error count to contribute to smaller WPMs. Controlling for uncorrected error rate, we indeed found a clear negative correlation between backspace count and WPM, $\beta=-0.61, p<.001$. Further, controlling also for glances at the text entry area, the effect remained at $\beta=-0.49, p<.001$.

\section{DISCUSSION}

A rich dataset was collected to deepen our understanding of how the fingers and eyes move in typing with mobile devices. The main finding over prior work is that movement strategies in mobile typing are strongly affected by competition for visual attention. Whereas with physical keyboards a skilled typist can keep his or her attention on the text display, where it is needed for detecting errors [29, 52], in mobile typing the need to guide finger motion competes for attention. Since one cannot monitor the keyboard and the text display at the same time, even though the mobile device is small, a strategy must be selected that determines which to give attention and when. A good strategy must strike a compromise between the cost of not correcting errors early and the time lost in glancing at the text display, when the fingers cannot be guided. Further, if the typist is not skilled with the keyboard, they need to conduct costly visual search, which we did not need to consider in our analysis [31]. Conversely, it is possible that a very skilled typist has learned to control finger movement to an extent that most of the time the gaze can be kept on the text area. However, a more detailed analysis of very fast typists would be required to investigate this. Supervisory control in mobile typing is, hence, not just about the speed-accuracy tradeoff of finger movement; at its core is the deployment of gaze between the main regions of the application. While cost-benefit analyses have shown this in the case of intelligent text-entry methods [50], the general point has not been made before with a support from data.

Understanding the competition for attention that goes on in typing helps us understand what makes typing fast vs. slow. It also makes important implications for smart typing aids, which in light of our results should not compete for attention and require learning of more complicated attention shift policies, like, for instance, word prediction lists do. We found that typing speed is positively correlated with the amount of attention on the touchscreen keyboard. The attention of a typical typist in our study was on the keyboard about $60 \%$ of the time, while the equivalent figure for a touch typist in a comparable study of physical keyboard typing was only $20 \%$ [16]. Also, the frequency of gaze shifts is much higher in mobile typing: 3.4 in our study, compared with 0.92 in the physical keyboard study. Similarities between mobile typing (our study) and 
typing on physical keyboards [16] include unequal division of labor between hands, the benefit of preparatory movements, and the negative effect of errors on typing speed. We further note that that study is similar to ours in key respects, including the task and the sample (our mean age 26, their 31 years).

The notion of resource competition can also help refine our understanding of the known superiority of two-thumb typing over one-finger typing $[3,47]$. It has been attributed to alternation between the lateral sides of the keyboard [45], and our results corroborate this. Switching between sides is faster than moving a finger from one side to another. But we also found that two-thumb-typing users benefit from preparatory movements, moving a soon-to-press finger toward its next target, similar to a pattern found in typing on physical keyboards [16]. However, significantly more errors are made when typing with two fingers instead of one. We found that in two-thumb typing, there are more intervening keypresses between consecutive glances at the text display. Users notice the errors later. Hence, the large benefits of two-finger use (shorter travel distance, preparatory movements) outweigh the costs (more delay in detection of errors). Making users aware of possible errors earlier presents an interesting challenge for intelligent text entry methods.

We also observed a curious and previously unreported phenomenon in two-thumb typing: there is unequal division of labor between the two lateral sides of the keyboard. Earlier models of two-thumb typing, based on log data rather than on direct observations of finger movement have assumed equal distribution [45]. We found, in contrast, that the right hand does most of the work. This was the dominant hand for most of the participants, but the same pattern was true for the lefthanded participants. The finger movement paths were significantly longer for the right (dominant-hand) finger than the left. While the monogram frequencies of Finnish might contribute to this, the effect has been observed also with non-Finnish typists using physical keyboards [16]. The unequal split between the hands could have implications for the customisation and adaptation of keyboard layouts.

Our results can inform the development of predictive models. The strong role of gaze deployment we found is in stark contrast with some previous accounts that have framed mobile typing in terms of finger movement [7, 62]. What analyses based on Fitts' law miss is the significant challenge posed to visual attention in typing: how to juggle between the two areas of the display that need attention. Fitts' law conceals these intriguing and critical effects in the empirical parameters ( $a$ and $b$ ). At the same, the existing non-Fittsian models similarly fail to account for the parallelism of gaze and finger movements. The KLM model of Holleis et al. [24], its extension by Sarcar et al. [56], and the ACT-R model of Cao et al. [9] all assume that either the gaze or the finger is moving but not both. Finally, it is important to develop generative models that model how eye-hand strategies adapt - for example, to changing probability of errors, to the number of fingers used, to the cost of error correction, and with time/experience. While these points have been made before $[31,56,50]$, we currently lack a unified model. In light of our findings, such a model must be able to explicate the role of attention control. We propose that hierarchical reinforcement learning [8] is a potential candidate control principle to explain thse gaze deployment strategies

\section{LIMITATIONS AND FUTURE WORK}

A few caveats must be taken into account when interpreting our findings. Firstly, our experiment was conducted in a quiet laboratory, with participants comfortably seated and resting their arms on the table. Mobile typing often takes place in dynamic environments, and there might be substantial differences in performance and strategies $[46,51]$. The instrumentation we used in our study cannot be easily used in the wild, but it is possible to design laboratory interventions which emulate reallife circumstances, such as walking or multitasking (e.g,. [6]). Second, our participants used a normal touchscreen Qwerty keyboard without intelligent text-entry aids, such as error correction or word prediction. As most of smartphone users seem to be using intelligent aids [47], studying this phenomenon is an important future work.

Third, we asked the users to correct all errors, a practice followed in some but not all text-entry studies. On one hand, this simplifies typing, since one need not regulate which errors are to be left as-is and which not, but, on the other, this renders it more important for the user to check the text display, because errors must not be left uncorrected. We believe that our main finding will not fundamentally change with the introduction of mobility, intelligent aids, or errors, but these could result in different attention sharing strategies. We believe that these factors complicate the problem that cognition faces in typing, simply because there are more tasks competing for visual attention [46]. Thus we expect even larger variability in movement strategies and, consequently, in typing performance. Fourth, our participants were relatively young adults with experience of technology, which is important to keep in mind since, for instance, older adults are generally slower at typing on smartphones [58]. A study with wider participant demographics is warranted for the future. The final caveat involves the language in the experiment. While we used a standard mobile corpus [59], the sentences were translated into Finnish, which has unique n-gram distributions and grammar. The keyboard layout we used also had two additional umlaut characters to the right. We do not expect the role of visual attention as reported here to differ greatly because of language, but the finger movement paths may vary between languages.

\section{CONCLUSION}

In this paper, we report rich and detailed finger and eye movement data from mobile typing. We illustrate and discuss the role of visual attention in mobile typing, contrasting it to typing with physical keyboards. To facilitate further research on this topic, we have made the software and analysis scripts, along with all data and instructions on how to analyze it at https: //userinterfaces . aalto . fi/how-we-type-mobile/.

\section{ACKNOWLEDGMENTS}

The research was supported by the Academy of Finland (grant numbers 291556, 310947) and the European Research Council (grant number 637991). 


\section{REFERENCES}

[1] John R. Anderson, Lynne M. Reder, and Christian Lebiere. 1996. Working Memory: Activation Limitations on Retrieval. Cognitive Psychology 30, 3 (June 1996), 221-256. DOI : http://dx.doi.org/10.1006/cogp. 1996.0007

[2] Ahmed Sabbir Arif, Sunjun Kim, Wolfgang Stuerzlinger, Geehyuk Lee, and Ali Mazalek. 2016. Evaluation of a Smart-Restorable Backspace Technique to Facilitate Text Entry Error Correction. In Proceedings of the 2016 CHI Conference on Human Factors in Computing Systems (CHI '16). ACM, New York, NY, USA, 5151-5162. DOI : http://dx.doi.org/10.1145/2858036.2858407

[3] Shiri Azenkot and Shumin Zhai. 2012. Touch Behavior with Different Postures on Soft Smartphone Keyboards. In Proceedings of the 14th International Conference on Human-computer Interaction with Mobile Devices and Services (MobileHCI '12). ACM, New York, NY, USA, 251-260. DOI :

http://dx.doi.org/10.1145/2371574.2371612

[4] Dana H Ballard, Mary M Hayhoe, Feng Li, and Steven D Whitehead. 1992. Hand-eye coordination during sequential tasks. Philosophical Transactions of the Royal Society of London. Series B: Biological Sciences 337, 1281 (1992), 331-339. DOI: http://dx.doi.org/10.1098/rstb.1992.0111

[5] Nikola Banovic, Varun Rao, Abinaya Saravanan, Anind K Dey, and Jennifer Mankoff. 2017. Quantifying Aversion to Costly Typing Errors in Expert Mobile Text Entry. In Proceedings of the 2017 CHI Conference on Human Factors in Computing Systems. ACM, 4229-4241. DOI :

http://dx.doi.org/10.1145/3025453.3025695

[6] Joanna Bergstrom-Lehtovirta, Antti Oulasvirta, and Stephen Brewster. 2011. The effects of walking speed on target acquisition on a touchscreen interface. In Proceedings of the 13th International Conference on Human Computer Interaction with Mobile Devices and Services. ACM, 143-146.

[7] Xiaojun Bi, Yang Li, and Shumin Zhai. 2013. FFitts Law: Modeling Finger Touch with Fitts' Law. In Proceedings of the SIGCHI Conference on Human Factors in Computing Systems (CHI '13). ACM, New York, NY, USA, 1363-1372. DOI : http://dx.doi.org/10.1145/2470654.2466180

[8] Matthew Michael Botvinick. 2012. Hierarchical reinforcement learning and decision making. Current Opinion in Neurobiology 22, 6 (2012), 956-962.

[9] Shi Cao, Anson Ho, and Jibo He. 2018. Modeling and predicting mobile phone touchscreen transcription typing using an integrated cognitive architecture. International Journal of Human-Computer Interaction 34, 6 (2018), 544-556. DOI :

http://dx.doi.org/10.1080/10447318.2017.1373463
[10] Tania Cerni, Marieke Longcamp, and Remo Job. 2016. Two thumbs and one index: A comparison of manual coordination in touch-typing and mobile-typing. Acta Psychologica 167 (June 2016), 16-23. DOI: http://dx.doi.org/10.1016/j. actpsy.2016.03.007

[11] Mary J. Chapman, Alice F. Healy, and James A. Kole. 2016. Memory load as a cognitive antidote to performance decrements in data entry. Memory 24, 9 (2016), 1182-1196. DOI :

http://dx.doi.org/10.1080/09658211.2015.1086380 PMID: 26390366.

[12] Edward Clarkson, Kent Lyons, James Clawson, and Thad Starner. 2007. Revisiting and Validating a Model of Two-thumb Text Entry. In Proceedings of the SIGCHI Conference on Human Factors in Computing Systems (CHI '07). ACM, New York, NY, USA, 163-166. DOI : http://dx.doi.org/10.1145/1240624.1240650

[13] James W Cortada. 2000. Before the computer: IBM, NCR, Burroughs, and Remington Rand and the industry they created, 1865-1956. Princeton University Press.

[14] Vivek Dhakal, Anna Maria Feit, Per Ola Kristensson, and Antti Oulasvirta. 2018. Observations on Typing from 136 Million Keystrokes. In Proceedings of the 2018 CHI Conference on Human Factors in Computing Systems (CHI '18). ACM, New York, NY, USA, Article 646,12 pages. DOI :

http://dx.doi.org/10.1145/3173574.3174220

[15] M.D. Dunlop and M. Montgomery Masters. 2009. Pickup usability dominates: a brief history of mobile text entry research and adoption. International Journal of Mobile Human Computer Interaction 1, 1 (2009), 42-59.

[16] Anna Maria Feit, Daryl Weir, and Antti Oulasvirta. 2016. How We Type: Movement Strategies and Performance in Everyday Typing. In Proceedings of the 2016 CHI Conference on Human Factors in Computing Systems (CHI '16). ACM, New York, NY, USA, 4262-4273. DOI : http://dx.doi.org/10.1145/2858036.2858233

[17] Paul M Fitts. 1954. The information capacity of the human motor system in controlling the amplitude of movement. Journal of experimental psychology 47, 6 (1954), 381. DOI : http://dx.doi.org/10.1037/h0055392

[18] Samuel J Gershman, Eric J Horvitz, and Joshua B Tenenbaum. 2015. Computational rationality: A converging paradigm for intelligence in brains, minds, and machines. Science 349, 6245 (2015), 273-278. DOI : http://dx.doi.org/10.1126/science. aac6076

[19] John D Gould, Sharon L Greene, Stephen J Boies, Antonia Meluson, and Marwan Rasamny. 1990. Using a touchscreen for simple tasks. Interacting with computers 2, 1 (1990), 59-74.

[20] Patricia Marks Greenfield. 1984. Mind and Media: The Effects of Television, Video Games, and Computers. (1984). 
[21] Patricia M Greenfield, Patricia DeWinstanley, Heidi Kilpatrick, and Daniel Kaye. 1994. Action video games and informal education: Effects on strategies for dividing visual attention. Journal of applied developmental psychology 15, 1 (1994), 105-123. DOI: http://dx.doi .org/10.1016/0193-3973(94)90008-6

[22] Yves Guiard, Halla B. Olafsdottir, and Simon T. Perrault. 2011. Fitt's Law As an Explicit Time/Error Trade-off. In Proceedings of the SIGCHI Conference on Human Factors in Computing Systems (CHI '11). ACM, New York, NY, USA, 1619-1628. DOI :

http://dx.doi.org/10.1145/1978942.1979179

[23] Eve Hoggan, Stephen A. Brewster, and Jody Johnston. 2008. Investigating the Effectiveness of Tactile Feedback for Mobile Touchscreens. In Proceedings of the SIGCHI Conference on Human Factors in Computing Systems (CHI '08). ACM, New York, NY, USA, 1573-1582. DOI : http://dx.doi.org/10.1145/1357054.1357300

[24] Paul Holleis, Friederike Otto, Heinrich Hussmann, and Albrecht Schmidt. 2007. Keystroke-level Model for Advanced Mobile Phone Interaction. In Proceedings of the SIGCHI Conference on Human Factors in Computing Systems (CHI '07). ACM, New York, NY, USA, 1505-1514. DOI :

http://dx.doi.org/10.1145/1240624.1240851

[25] Christian Holz and Patrick Baudisch. 2011. Understanding Touch. In Proceedings of the SIGCHI Conference on Human Factors in Computing Systems (CHI '11). ACM, New York, NY, USA, 2501-2510. DOI : http://dx.doi.org/10.1145/1978942.1979308

[26] Marc Jeannerod. 1988. The neural and behavioural organization of goal-directed movements. Clarendon Press/Oxford University Press.

[27] Charles F. Jekel, Gerhard Venter, Martin P. Venter, Nielen Stander, and Raphael T. Haftka. 2019. Similarity measures for identifying material parameters from hysteresis loops using inverse analysis. International Journal of Material Forming 12, 3 (01 May 2019), 355-378. DOI :

http://dx.doi.org/10.1007/s12289-018-1421-8

[28] jkielty. 2019. Viewport, resolution, diagonal screen size and DPI for the most popular smartphones. (Jul 2019). https://deviceatlas.com/blog/viewport-resolution-dia gonal-screen-size-and-dpi-most-popular-smartphones

[29] Roger Johansson, Åsa Wengelin, Victoria Johansson, and Kenneth Holmqvist. 2010. Looking at the keyboard or the monitor: relationship with text production processes. Reading and Writing 23, 7 (01 Aug 2010), 835-851. DOI :

http://dx.doi.org/10.1007/s11145-009-9189-3

[30] Jussi Jokinen. 2017. Touch Screen Text Entry as Cognitively Bounded Rationality. In Proceedings of the Annual Cognitive Science Society Meeting.

[31] Jussi P. P. Jokinen, Sayan Sarcar, Antti Oulasvirta, Chaklam Silpasuwanchai, Zhenxin Wang, and Xiangshi
Ren. 2017. Modelling Learning of New Keyboard Layouts. In Proceedings of the 2017 CHI Conference on Human Factors in Computing Systems (CHI '17). ACM, New York, NY, USA, 4203-4215. DOI: http://dx.doi.org/10.1145/3025453.3025580

[32] Charles M Judd, Jacob Westfall, and David A Kenny. 2012. Treating stimuli as a random factor in social psychology: A new and comprehensive solution to a pervasive but largely ignored problem. Journal of personality and social psychology 103, 1 (2012), 54. DOI : http://dx.doi.org/10.1037/a0028347

[33] Kevin S. Killourhy and Roy A. Maxion. 2012. Free vs. Transcribed Text for Keystroke-dynamics Evaluations. In Proceedings of the 2012 Workshop on Learning from Authoritative Security Experiment Results (LASER '12). ACM, New York, NY, USA, 1-8. DOI:

http://dx.doi.org/10.1145/2379616.2379617

[34] Per Ola Kristensson. 2009. Five challenges for intelligent text entry methods. AI Magazine 30, 4 (2009), 85-85. DOI :

http://dx.doi.org/10.1609/aimag.v30i4.2269

[35] Tuomo Kujala, Jakke Mäkelä, Ilkka Kotilainen, and Timo Tokkonen. 2016. The attentional demand of automobile driving revisited: occlusion distance as a function of task-relevant event density in realistic driving scenarios. Human factors 58, 1 (2016), 163-180. DOI : http://dx.doi.org/10.1177/0018720815595901

[36] Richard L Lewis, Andrew Howes, and Satinder Singh. 2014. Computational rationality: Linking mechanism and behavior through bounded utility maximization. Topics in cognitive science 6, 2 (2014), 279-311. DOI: http://dx.doi.org/10.1111/tops. 12086

[37] Yilin Li, Baochang Zhang, Yao Cao, Sanqiang Zhao, Yongsheng Gao, and Jianzhuang Liu. 2011. Study on the BeiHang Keystroke Dynamics Database. In 2011 International Joint Conference on Biometrics (IJCB). IEEE. DOI :

http://dx.doi.org/10.1109/ijcb. 2011.6117485

[38] Gordon D. Logan. 2018. Automatic control: How experts act without thinking. Psychological Review 125, 4 (July 2018), 453-485. DOI :

http://dx.doi .org/10.1037/rev0000100

[39] Gordon D Logan and Matthew JC Crump. 2011. Hierarchical control of cognitive processes: The case for skilled typewriting. In Psychology of learning and motivation. Vol. 54. Elsevier, 1-27. DOI :

http://dx. doi .org/10.1016/b978-0-12-385527-5.00001-2

[40] Yiqin Lu, Chun Yu, Shuyi Fan, Xiaojun Bi, and Yuanchun Shi. 2019. Typing on Split Keyboards with Peripheral Vision. In Proceedings of the 2019 CHI Conference on Human Factors in Computing Systems (CHI '19). ACM, New York, NY, USA, Article 200, 12 pages. DOI :

http://dx.doi .org/10.1145/3290605.3300430 
[41] I Scott MacKenzie and R William Soukoreff. 2002a. A model of two-thumb text entry. In Graphics interface. 117-124.

[42] I Scott MacKenzie and R William Soukoreff. 2002b. Text entry for mobile computing: Models and methods, theory and practice. Human-Computer Interaction 17, 2-3 (2002), 147-198.

[43] John A Michon. 1985. A critical view of driver behavior models: what do we know, what should we do? In Human behavior and traffic safety. Springer, 485-524.

[44] Urpo Nikanne. 2017. Finite Sentences In Finnish: Word Order, Morphology, And Information Structure. (2017). DOI : http://dx.doi.org/10.5281/zenodo. 1117710

[45] Antti Oulasvirta, Anna Reichel, Wenbin Li, Yan Zhang, Myroslav Bachynskyi, Keith Vertanen, and Per Ola Kristensson. 2013. Improving Two-thumb Text Entry on Touchscreen Devices. In Proceedings of the SIGCHI Conference on Human Factors in Computing Systems (CHI '13). ACM, New York, NY, USA, 2765-2774. DOI : http://dx.doi.org/10.1145/2470654.2481383

[46] Antti Oulasvirta, Sakari Tamminen, Virpi Roto, and Jaana Kuorelahti. 2005. Interaction in 4-second Bursts: The Fragmented Nature of Attentional Resources in Mobile HCI. In Proceedings of the SIGCHI Conference on Human Factors in Computing Systems (CHI'05). ACM, New York, NY, USA, 919-928. D0I: http://dx.doi.org/10.1145/1054972.1055101

[47] Kseniia Palin, Anna Feit, Sunjun Kim, Per Ola Kristensson, and Antti Oulasvirta. 2019. How do People Type on Mobile Devices? Observations from a Study with 37,000 Volunteers. In Proceedings of 21st International Conference on Human-Computer Interaction with Mobile Devices and Services (MobileHCI'19). ACM. DOI :

http://dx.doi.org/https://doi.org/10.475/123_4

[48] Alexandra Papoutsaki, Aaron Gokaslan, James Tompkin, Yuze He, and Jeff Huang. 2018. The Eye of the Typer: A Benchmark and Analysis of Gaze Behavior During Typing. In Proceedings of the 2018 ACM Symposium on Eye Tracking Research \& Applications (ETRA '18). ACM, New York, NY, USA, Article 16, 9 pages. DOI : http://dx.doi.org/10.1145/3204493. 3204552

[49] Ken Pfeuffer and Yang Li. 2018. Analysis and Modeling of Grid Performance on Touchscreen Mobile Devices. In Proceedings of the 2018 CHI Conference on Human Factors in Computing Systems (CHI'18). ACM, New York, NY, USA, Article 288, 12 pages. DOI : http://dx.doi.org/10.1145/3173574. 3173862

[50] Philip Quinn and Shumin Zhai. 2016. A Cost-Benefit Study of Text Entry Suggestion Interaction. In Proceedings of the 2016 CHI Conference on Human Factors in Computing Systems (CHI'16). ACM, New York, NY, USA, 83-88. DOI :

http://dx.doi.org/10.1145/2858036.2858305
[51] Shyam Reyal, Shumin Zhai, and Per Ola Kristensson. 2015. Performance and user experience of touchscreen and gesture keyboards in a lab setting and in the wild. In Proceedings of the 33rd Annual ACM Conference on Human Factors in Computing Systems. ACM, 679-688.

[52] Martina Rieger and Victoria Bart. 2016. Typing Style and the Use of Different Sources of Information during Typing: An Investigation Using Self-Reports. Frontiers in Psychology 7 (12 2016), 1-12. DOI: http://dx.doi.org/10.3389/fpsyg.2016.01908

[53] Uta Sailer, J Randall Flanagan, and Roland S Johansson. 2005. Eye-hand coordination during learning of a novel visuomotor task. Journal of Neuroscience 25, 39 (2005), 8833-8842. DOI :

http://dx.doi.org/10.1523/jneurosci.2658-05.2005

[54] Timothy A. Salthouse. 1984. Effects of age and skill in typing. Journal of Experimental Psychology: General 113, 3 (1984), 345-371. DOI :

http://dx.doi .org/10.1037/0096-3445.113.3.345

[55] Timothy A. Salthouse. 1986. Perceptual, cognitive, and motoric aspects of transcription typing. Psychological Bulletin 99, 3 (1986), 303-319. DOI :

http://dx.doi.org/10.1037/0033-2909.99.3.303

[56] Sayan Sarcar, Jussi PP Jokinen, Antti Oulasvirta, Zhenxin Wang, Chaklam Silpasuwanchai, and Xiangshi Ren. 2018. Ability-based optimization of touchscreen interactions. IEEE Pervasive Computing 17, 1 (2018), 15-26. DOI :

http://dx.doi.org/10.1109/mprv.2018.011591058

[57] Andrew Sears, Julie A Jacko, Josey Chu, and Francisco Moro. 2001. The role of visual search in the design of effective soft keyboards. Behaviour \& Information Technology 20, 3 (2001), 159-166. DOI : http://dx.doi .org/10.1080/01449290110049790

[58] Amanda L Smith and Barbara S Chaparro. 2015. Smartphone text input method performance, usability, and preference with younger and older adults. Human factors 57, 6 (2015), 1015-1028. DOI : http://dx.doi .org/10.1177/0018720815575644

[59] Keith Vertanen and Per Ola Kristensson. 2011. A versatile dataset for text entry evaluations based on genuine mobile emails. In Proceedings of the 13th International Conference on Human Computer Interaction with Mobile Devices and Services. ACM, ACM Press, 295-298. DOI : http://dx.doi.org/10.1145/2037373.2037418

[60] Pierre Weill-Tessier and Hans Gellersen. 2018. Touch Input and Gaze Correlation on Tablets. In Intelligent Decision Technologies 2017, Ireneusz Czarnowski, Robert J. Howlett, and Lakhmi C. Jain (Eds.). Springer International Publishing, Cham, 287-296.

[61] Pierre Weill-Tessier, Jayson Turner, and Hans Gellersen. 2016. How Do You Look at What You Touch?: A Study of Touch Interaction and Gaze Correlation on Tablets. In Proceedings of the Ninth Biennial ACM Symposium on 
Eye Tracking Research \& Applications (ETRA '16). ACM, New York, NY, USA, 329-330. DOI: http://dx.doi.org/10.1145/2857491.2888592

[62] R William Soukoreff and I Scott Mackenzie. 1995. Theoretical upper and lower bounds on typing speed using a stylus and a soft keyboard. Behaviour \& Information Technology 14, 6 (1995), 370-379. DOI : http://dx.doi .org/10.1080/01449299508914656

[63] Katharina Witowski and Nielen Stander. 2012. Parameter Identification of Hysteretic Models Using Partial Curve Mapping. In 12th AIAA Aviation Technology, Integration, and Operations (ATIO) Conference and 14th AIAA/ISSMO Multidisciplinary
Analysis and Optimization Conference. American Institute of Aeronautics and Astronautics. DOI : http://dx. doi .org/10.2514/6.2012-5580

[64] Jacob O. Wobbrock. 2007. Measures of Text Entry Performance. In Text Entry Systems. Elsevier, 47-74. DOI :

http://dx. doi .org/10.1016/b978-012373591-1/50003-6

[65] Motonori Yamaguchi and Gordon D. Logan. 2014. Pushing typists back on the learning curve: Revealing chunking in skilled typewriting. Journal of Experimental Psychology: Human Perception and Performance 40, 2 (2014), 592-612. DOI :

http://dx.doi.org/10.1037/a0033809 